\title{
Quality of experience issues in multimedia provision
}

\author{
Harilaos Koumaras · Fidel Liberal · Lingfen Sun
}

Published online: 8 June 2010

(C) Springer Science+Business Media, LLC 2010

Currently, the success of providing novel multimedia services over wired/wireless networks depends on how good the quality of the service is and whether it meets an end user's expectations. Thus, it is critical for equipment manufacturers, network operators and service providers to be able to assess, predict and possibly control the end-to-end perceptual multimedia (e.g., voice and video) quality for commercial and technical reasons.

The Quality of Service (QoS) perceived as user satisfaction has received through past years some efforts from the research community, introducing the concept of the Quality of Experience (QoE). The evaluation of the QoE will provide an end user with a range of potential choices, covering the possibilities of low, medium, or high quality levels. This QoE evaluation will also give service providers and network operators the capability to minimize the storage and network resources by allocating only the ones that are necessary to maintain a specific level of user satisfaction.

This special issue focuses on novel accurate, efficient and robust QoE models for multimedia services, including both VoIP and video services, novel QoE-driven cross-layer architectures, including performance evaluation, traffic man-

H. Koumaras $(\bowtie)$

Institute of Informatics and Telecommunications, NCSR

Demokritos, Athens, Greece

e-mail: koumaras@iit.demokritos.gr

F. Liberal

Department of Electronics and Telecommunications, University

of the Basque Country (UPV/EHU), Bilbao, Spain

e-mail: fidel.liberal@ehu.es

L. Sun

School of Computing, Communications and Electronics,

University of Plymouth, Plymouth PL4 8AA, UK

e-mail: L.Sun@plymouth.ac.uk agement and cross-layer solutions. More specifically, this special issue consists of 11 research papers, which have been classified into three Sections. Sections 1 and 2 both consist of papers that deal with QoE estimation models in VoIP and Video services respectively, while Section 3 deals with QoEdriven Adaptation and Management schemes. More specifically:

- Section 1 is devoted to VoIP Quality Assessment Models, consisting of 2 papers that present, evaluate and demonstrate novel estimation models.

In the first paper of this section, Maria-Dolores Cano and Fernando Cerdan carry out a subjective QoE assessment for VoIP applications in a real wireless environment. Skype, Gizmo5, ooVoo, and Damaka are the selected VoIP applications under study. Afterwards, the authors compare QoE results with QoS evaluation from the captured video calls made during the poll. The paper results show that there is no precise match on both assessments, because of the QoE parameters that cannot be inferred from QoS analysis, due to the different effect of some QoS parameters which prevail, or even because of the popularity of the VoIP application.

In the second paper of the VoIP section, Sofiene Jelassi, Habib Youssef, Christian Hoene and Guy Pujolle propose novel no-reference parametric speech quality estimate models, which account for the voicing feature of signal wave included in missing packets. The authors develop separate speech quality estimate models, which capture the perceptual effect of the lost voiced or unvoiced packets, using elaborated simple and multiple regression analyses. A new speech quality estimate model, which mixes voiced and unvoiced quality scores to compute the overall speech quality score at the end of an assessment interval, is proposed following a rigorous multiple linear regression analysis. 
- Section 2 deals with the Video Quality Assessment Models, consisting of 4 papers that present, evaluate and demonstrate novel estimation and prediction models.

The first paper of this section, authored by Quan Huynh-Thu and Mohammed Ghanbari, examines the accuracy of PSNR in predicting video quality for different video scenes and frame rates, the authors show that PSNR follows a monotonic relationship with subjective quality in the case of full frame rate encoding when the video content and codec are fixed. More specifically, it is shown that PSNR is inaccurate in measuring video quality of a video content encoded at different frame rates because it is not capable of assessing the perceptual trade-off between the spatial and temporal qualities.

In the second paper of this section, Jose Joskowicz and J. Carlos Lopez Ardao propose a novel parametric model, which provides estimation for the perceived quality of video, coded with different codecs, at any bit rate and display format. The validity of the proposed model is supported by an experimental set of more than 1500 processed video clips, coded in MPEG2 and H.264/AVC, in various bit rates and display formats.

The third paper of this section, authored by Xiaodong Gu, Guoping Qiu, Xu Feng, Liu Debing, Chen Zhibo presents a region of interest weighted pooling strategy for video quality, considering the influence of visual attention. Apparent and coherent performance improvement is achieved by applying the proposed pooling strategy on PSNR and SSIM, together with a highly reduction in computation complexity.

In the fourth paper of this section, the authors Georgios Gardikis, George Xilouris, Evangelos Pallis and Anastasios Kourtis present a joint assessment of network- and perceived-QoS in video delivery networks, with the aim of correlating these two parameters for a specific network and service configuration. This framework/architecture is implemented with open source software tools and has been demonstrated in an actual WiMAX streaming video distribution platform.

- Section 3 deals with QoE-driven Adaptation and Management Schemes for both Video and VoIP services, consisting of five papers.

The first paper of this section, authored by Florence Agboma and Antonio Liotta contributes towards the relatively new but growing discipline of QoE management in content delivery systems. The paper focuses on the development of a QoE-based management framework for the construction of QoE models for different types of multimedia contents delivered onto three typical mobile terminals-a mobile phone, PDA and a laptop. A statistical modelling technique is employed which, correlates QoS parameters with estimates of QoE perceptions.

In the second paper of this section, the authors E. Jammeh, I. Mkwawa, A. Khan, M. Goudarzi, L. Sun and E. Ifeachor present a QoE-driven adaptation scheme for voice and video over IP to solve the optimization problem to provide optimal QoE for networked voice and video applications. The adaptive VoIP architecture was implemented and tested both in NS2 and in an Open IMS Core network to allow extensive simulation and test-bed evaluation. Results show that the scheme was optimally responsive to available network bandwidth and congestion for both voice and video and optimised delivered QoE for different network conditions, and is friendly to TCP traffic.

The third paper, authored by Jânio M. Monteiro, Carlos T. Calafate, Mário S. Nunes, evaluates the quality enhancements introduced by the integration of several H.264/SVC layers with a Raptor coding protection scheme. The aim of the paper is to improve the distribution of video over loss prone networks in terms of ratedistortion performance by assessing several alternative packetization options and protection schemes.

In the fourth paper, the authors Chris Develder et al. propose an architecture combining a real-time video quality monitoring platform in a heterogeneous home network based on UPnP QoS v3. From a research perspective, the authors propose a new subjective test procedure that revealed user preference for temporal scalability over quality scalability.

Finally, the fifth paper of this section, authored by Zhenyu Na, Qing Guo, Zihe Gao, Jiaqi Zhen, Changyu Wang, presents a novel adaptive traffic prediction AQM (ATPAQM) algorithm, operating in two granularities. In coarse granularity, it adopts an improved Kalman filtering model to predict traffic and calculates average packet loss ratio (PLR) at every prediction interval. In fine granularity, upon receiving a packet, it regulates packet dropping probability according to the calculated average PLR. Simulation results show that the proposed algorithm outperforms other algorithms in queue stability, packet loss ratio and link utilization.

The guest editors would like to thank all the authors of the papers that selected this special issue in order to publish their research results. We are also grateful to all our reviewers for their constructive and helpful comments and suggestions to help us to keep the quality high. We have the feeling that this special issue has successfully accomplished its scope by contributing a small piece in the research community of QoE issue. 


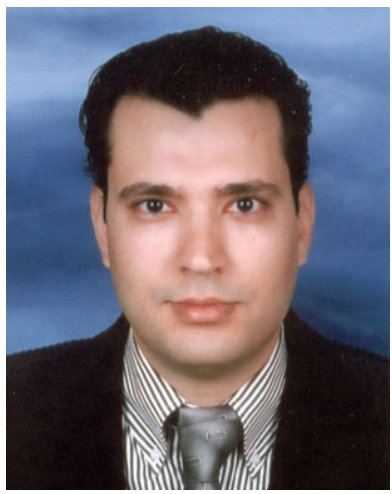

Harilaos Koumaras was born in Athens, Greece in 1980. He received his B.Sc. degree in Physics in 2002 from the University of Athens, Physics Department, his M.Sc. in Electronic Automation and Information Systems in 2004, being scholar of the non-profit organization Alexander S Onassis, from the University of Athens, Physics and Informatics Department and his $\mathrm{Ph} . \mathrm{D}$. in 2007 on digital video quality prediction from the University of Athens, Informatics Department, having granted the four-year scholarship of NCSR "Demokritos". He has received twice the Greek State Foundations (IKY) scholarship during the academic years 2000-01 and 2003-04. He has also granted with honors the classical piano and harmony degrees from the classical music department of Attiko Conservatory. He joined the Digital Telecommunications Lab at the National Centre of Scientific Research "Demokritos" in 2003 and since then he has participated in EU-funded and national funded projects with presentations and publications at international conferences, scientific journals and book chapters. At the same time, he is an associate lecturer at the Business College of Athens (BCA) and City University of Seattle, teaching modules related to Information Technology, Data Networks and Mathematics. His research interests include objective/subjective evaluation of the perceived quality of multimedia services, video quality and picture quality evaluation, video traffic modeling, digital terrestrial television and video compression techniques. Currently, he is the author or co-author of more than 40 scientific papers in international journals, technical books and book chapters, numbering 75 non-self citations. He is an editorial board member of Telecommunications Systems Journal and a reviewer of EURASIP Journal of Applied Signal Processing and IEEE Transactions on Broadcasting. Dr. Koumaras is a member of IEEE, SPIE and National Geographic Society.

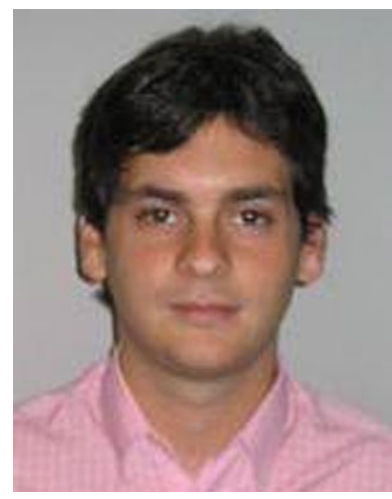

Fidel Liberal received the B.Sc. and M.Sc. degrees in Telecommunications Engineering from the University of the Basque Country, Spain, in 2001. In 2005 he received the $\mathrm{Ph} . \mathrm{D}$. in Telecommunications Engineering from the same University for his work in the area of holistic management of quality (both NQoS and PQoS-QoE) in telecommunications services. He currently works as a lecturer in the Faculty of Engineering of Bilbao and cooperates in different national and European R\&D projects. His research interests include QoS and QoE management and multicriteria optimization in MANETs and NGNs.

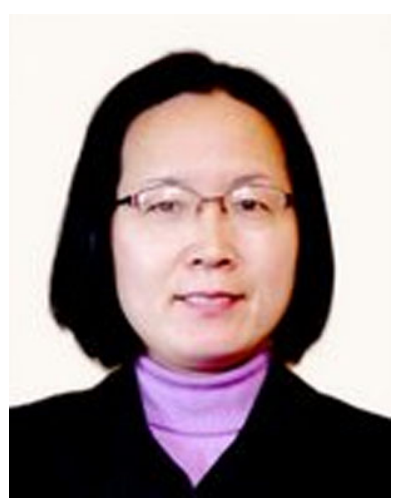

Lingfen Sun received her Ph.D. degree in VoIP speech quality prediction from the University of Plymouth UK in 2004. She holds a M.Sc. in Communication and Electronics System (1988) and B.Eng. in Telecommunications Engineering (1985) from the Institute of Communications Engineering, Nanjing, China. She is now a Lecturer in Computer Networks in the School of Computing, Communications and Electronics, University of Plymouth, UK. She has been involved in several government/industry funded projects on multimedia communications \& networking and leads group's research in this area. She has published over 40 papers in peer-refereed journals and conference proceedings. Her publications on VoIP speech quality have received more than 150 citations by peer researchers. She is a reviewer for journals such as IEEE Transactions on Multimedia, IET Electronics Letters and IEEE Transactions on Speech and Audio Processing. She has served on the technical programme committees (TPCs) of a number of international conferences, including IEEE Globecom, Accessnets and Chinacom. Her main research interests include VoIP, QoS, voice/video quality assessment (objective and subjective), quality of service prediction and control for multimedia over packet, mobile and wireless networks, network performance measurement and characterisation, and multimedia quality management. She is a member of IEEE and a Voting Member of IEEE Multimedia Communication Technical Committee. 